\title{
Variance-Constrained Robust Estimation for Discrete-Time Systems with Communication Constraints
}

\author{
Baofeng Wang, ${ }^{1,2}$ Ge Guo, ${ }^{2}$ and Xiue Gao ${ }^{1}$ \\ ${ }^{1}$ Information Engineering Institute, Dalian University, Dalian 116622, China \\ ${ }^{2}$ School of Information Science and Technology, Dalian Maritime University, Dalian 116026, China \\ Correspondence should be addressed to Baofeng Wang; wangbaofeng@dlu.edu.cn
}

Received 6 September 2013; Accepted 22 December 2013; Published 14 January 2014

Academic Editor: Fuzhong Nian

Copyright (c) 2014 Baofeng Wang et al. This is an open access article distributed under the Creative Commons Attribution License, which permits unrestricted use, distribution, and reproduction in any medium, provided the original work is properly cited.

\begin{abstract}
This paper is concerned with a new filtering problem in networked control systems (NCSs) subject to limited communication capacity, which includes measurement quantization, random transmission delay, and packets loss. The measurements are first quantized via a logarithmic quantizer and then transmitted through a digital communication network with random delay and packet loss. The three communication constraints phenomena which can be seen as a class of uncertainties are formulated by a stochastic parameter uncertainty system. The purpose of the paper is to design a linear filter such that, for all the communication constraints, the error state of the filtering process is mean square bounded and the steady-state variance of the estimation error for each state is not more than the individual prescribed upper bound. It is shown that the desired filtering can effectively be solved if there are positive definite solutions to a couple of algebraic Riccati-like inequalities or linear matrix inequalities. Finally, an illustrative numerical example is presented to demonstrate the effectiveness and flexibility of the proposed design approach.
\end{abstract}

\section{Introduction}

State estimation has long been a significant interesting problem in the control and information fields. In recent years, with technological advances in MEMS, DSP capabilities, computing, and communication technology, networked control systems (NCSs) are built massively, in which sensors, actuators, and controllers are not physically collocated [1, 2]. Hence, there are various uncertainties caused by limited communication capacity when exchanging information via a digital communication network [3-5]. With NCSs becoming more and more popular in various applications, state estimation problem with limited communication capacity has attracted recurring interests. Generally, there are three important issues that should be addressed for communicationbased systems, that is, signal transmission delay, packet loss, and quantization effect. For example, the packet loss has been described by the binary Bernoulli distribution approach $[6,7]$ as well as Markovian jump approach [8], packet delay has been investigated as constant delay [9] and bounded delay [5] along with unbounded packet delay [10], and the quantization has been dealt with via a sector bound approach where the quantization errors are regarded as a class of uncertainties [11]. However, the aforementioned pieces of literature still suffer from some limitations such as the fact that most results only investigate one or two aspects of the communication issues mentioned above, especially quantization effect has been paid little attention in network-based system; some papers are concentrated on controller design problem, while state estimation problem has been neglected, which is equally important in applications and so on.

For networked environment, much effort has been made towards proposing useful alternative methods in different contexts $[6,8,10,12-15]$, among which $H_{\infty}$ filtering and robust filtering approaches are prominent to improve the robustness. Generally, there are two popular approaches used to design a robust filter, that is, Riccati equations approach $[16,17]$ and linear matrix inequalities approach [18]. However, in some practical engineering such as the tracking of a maneuvering target, the filtering performance requirements are described as the upper bounds in the error variances of estimation, that is, the steady-state error variance is not required to be the minimum but should not be more than 
the specified upper bound constraint. Based on the error covariance assignment (ECA) theory [19], which provided a closed form solution for directly placing the specified steadystate estimation error covariance to a linear system, varianceconstrained filtering method has been developed and applied to some more realistic system. For example, sampled-data system was studied in [20] and bilinear systems in [21]. In $[7,22]$, Wang et al. considered the filtering problem for linear uncertain discrete-time system with missing measurements and randomly varying sensor delay, respectively. However, quantization effect and transmission delay or packet dropouts were neglected.

In this paper, we investigate the filtering problem for discrete-time system in networked control systems (NCSs) subjected to limited communication capacity including the three aspects previously discussed. Through transmitting the communication limitations into a stochastic uncertainty system representation, variance-constrained filter problems are considered. It is shown that the filtering can effectively be derived if there are positive definite solutions to a couple of algebraic Riccati-like inequalities or linear matrix inequalities. Relative to the traditional optimal robust or $H_{\infty}$ filtering, for variance-constrained filtering approach, there still remains much freedom to be used for achieving other desired performance requirements after assigning to the system a specified variance upper bound. An illustrative numerical example is used to demonstrate the usefulness and flexibility of the proposed design approach.

The rest of this paper is arranged as follows. In Section 2, the robust filter design problem for discrete-time systems with communication limitations is formulated. Some preliminary results are given in Section 3, and the solution of this problem is developed in Section 4. In Section 5, the effectiveness of the proposed theory is demonstrated by an illustrative example. Some concluding remarks and future research directions are draw in Section 6.

\section{Problem Formulation and Preliminaries}

Consider the following linear discrete-time stochastic system:

$$
\begin{gathered}
\tilde{x}(k+1)=\widetilde{A} \tilde{x}(k)+\widetilde{B} w(k), \\
z(k)=\widetilde{C} \tilde{x}(k)+\widetilde{v}(k),
\end{gathered}
$$

where $\tilde{x}(k) \in R^{n}$ is the state vector, $z(k) \in R^{m}$ is the measured output vector, and $w(k) \in R^{n}$ and $\widetilde{v}(k) \in R^{m}$ are the process noise and measurement noise, respectively. $\widetilde{A}, \widetilde{C}$, and $\widetilde{B}$ are known real constant matrices with appropriate dimensions.

We make the following assumptions on the statistical properties of the initial state, process noise, and measurement noise, which are also standard in Kalman filtering.

Assumption 1. For all integers $k, l \geq 0$,

(1) $E\{\tilde{x}(0)\}=E\{w(k)\}=E\{\widetilde{v}(k)\}=0$,

(2) $E\left\{\tilde{x}(0) \tilde{x}^{T}(0)\right\}=\widetilde{P}(0)$,
(3)

$$
E\left\{\left[\begin{array}{l}
w(k) \\
\widetilde{v}(k)
\end{array}\right]\left[w^{T}(l) \widetilde{v}^{T}(l)\right]\right\}= \begin{cases}I_{n+m} & \text { for } k=l \\
0 & \text { for } k \neq l\end{cases}
$$

We point out that if $w(k)$ and/or $\widetilde{v}(k)$ are colored noises with known statistics, they can be prewhitened.

Furthermore, throughout the paper, the following assumption is made.

Assumption 2. The matrix $\widetilde{A}$ is Schur stable (i.e., all eigenvalues of $\widetilde{A}$ are located within the unit circle in the complex plane).

As aforementioned analysis, generally, three effects need to be taken into consideration: measurement quantization, packet transmission delays, and packet loss. We model them mathematically as follows.

(i) Measurement Quantization. It is assumed that the sampled measurements of $z(k)$ are first quantized via a quantizer, encapsulated into a packet, and then transmitted through a digital communication network. In this paper, we consider the logarithmic quantizer. According to $[3,23,24]$, the quantizer is denoted as $f(\cdot)=\left[\begin{array}{llll}f_{1}(\cdot) & f_{2}(\cdot) & \cdots & f_{p}(\cdot)\end{array}\right]$, which is symmetric; that is, $f_{j}(-v)=-f_{j}(v), j=1, \ldots, p$. For each $f_{j}(\cdot)$, the set of quantized levels is characterized by

$$
\begin{array}{r}
\Psi_{j}=\left\{ \pm \phi_{i}^{(j)}, \phi_{i}^{(j)}=\rho_{j}^{i} \phi_{0}^{(j)}, i= \pm 1, \pm 2, \ldots\right\} \cup\left\{ \pm \phi_{0}^{(j)}\right\} \cup\{0\} \\
0<\rho_{j}<1 ; \phi_{0}^{(j)}>0
\end{array}
$$

and the associated quantizer $f_{j}(\cdot)$ is defined as follows:

$$
f_{j}(v)= \begin{cases}\phi_{i}^{(j)}, & \text { if } \frac{1}{1+\sigma_{j}} \phi_{i}^{(j)}<v \leq \frac{1}{1-\sigma_{j}} \phi_{i}^{(j)}, v>0 \\ 0, & \text { if } v=0, \\ -f_{j}(-v), & \text { if } v<0,\end{cases}
$$

where

$$
\sigma_{j}=\frac{1-\rho_{j}}{1+\rho_{j}}
$$

Then, we have

$$
y^{q}(k)=f(z(k))=(I+\Lambda(k)) z(k),
$$

where $y^{q}(k)$ denotes the measurements after quantization, and

$$
\begin{gathered}
\Lambda(k)=\operatorname{diag}\left\{\Lambda_{1}(k), \Lambda_{2}(k), \ldots, \Lambda_{p}(k)\right\}, \\
\Lambda_{j}(k)=\left[-\sigma_{j}, \sigma_{j}\right], \quad j=1, \ldots, p .
\end{gathered}
$$


Furthermore, define

$$
\Lambda=\operatorname{diag}\left\{\sigma_{1}, \sigma_{2}, \ldots, \sigma_{m}\right\} .
$$

For convenience of later analysis, we make the following assumption:

$$
F(k)=\Lambda^{-1} \Lambda(k) .
$$

It is clear that $F(k)$ satisfies

$$
F^{T}(k) F(k) \leq I, \quad \forall k \in R .
$$

(ii) Randomly Varying Packet Delays and Dropouts. In the paper, we assume that the digital communication channel is subjected to the randomly bounded packet delays and dropouts, which is modeled as following

$$
y(k)=\gamma_{0}(k) y^{q}(k)+\left(1-\gamma_{0}(k)\right) \gamma_{1}(k) y^{q}(k-1),
$$

where $y(k)$ represents the system output subject to randomly varying delays and dropouts, and the stochastic variable $\gamma_{i} \epsilon$ $R(i \in 0,1)$ is a Bernoulli distributed white sequence taking values on 0 and 1 with following statistical properties.

Assumption 3. For stochastic variable $\gamma_{i} \in R(i \in 0,1)$, we have

(1) $\operatorname{Prob}\left\{\gamma_{i}=1\right\}=E\left\{\gamma_{i}\right\}:=\bar{\gamma}_{i}, \operatorname{Prob}\left\{\gamma_{i}=0\right\}=1-\bar{\gamma}_{i}$, where $\bar{\gamma}_{i} \in R(i \in 0,1)$ is a known positive scalar.

(2) $\gamma_{i}$ is independent of $\tilde{x}(0), w(k)$ and $\widetilde{v}(k)$.

(3) $\bar{\gamma}_{i} \in R(i \in 0,1)$ are mutually independent.

Directly, from (1), the following formula can be derived, $\sigma_{\gamma}^{2}=E\left\{\left(\gamma_{i}-\bar{\gamma}_{i}\right)^{2}\right\}=\left(1-\gamma_{i}\right) \bar{\gamma}_{i}$.

By substituting (6) into (11), we obtain

$$
\begin{aligned}
y(k)= & \gamma_{0}(k)(I+\Lambda(k)) z(k) \\
& +\gamma_{0}(k)\left(1-\gamma_{1}(k)\right)(I+\Lambda(k)) z(k-1) .
\end{aligned}
$$

And a compact representation of systems (1) and (12) can be given as follows:

$$
\begin{gathered}
x(k+1)=A x(k)+B w(k), \\
y(k)=\left(C_{\gamma(k)}+\Delta C_{\gamma(k)}(k)\right) x(k)+(D+\Delta D(k)) v(k),
\end{gathered}
$$

where $\Delta C_{\gamma(k)}(k)=\Lambda F(k) C_{\gamma(k)}, \Delta D(k)=\Lambda F(k) D$ and

$$
\begin{gathered}
x(k)=\left[\begin{array}{c}
\tilde{x}(k) \\
\tilde{x}(k-1)
\end{array}\right], \quad A=\left[\begin{array}{cc}
\widetilde{A} & 0 \\
I_{n} & 0
\end{array}\right], \\
B=\left[\begin{array}{c}
\widetilde{B} \\
0
\end{array}\right], \quad D=\left[\begin{array}{ll}
I_{m} & I_{m}
\end{array}\right], \\
C_{\gamma(k)}=\left[\begin{array}{c}
\gamma_{0}(k) \widetilde{C}\left(1-\gamma_{0}(k)\right) \gamma_{1}(k) \widetilde{C}
\end{array}\right], \\
v(k)=\left[\begin{array}{c}
\gamma_{0}(k) \widetilde{v}(k) \\
\left(1-\gamma_{0}(k)\right) \gamma_{1}(k) \widetilde{v}(k-1)
\end{array}\right] .
\end{gathered}
$$

For compact systems (13)-(14), observe that the matrices $C_{\gamma(k)}$ and $v(k)$ contain stochastic parameter $\gamma(k)$; in view of Assumptions 1 and 3, we have Lemma 4.
Lemma 4. For systems (13) and (14), the initial state $x(0)$ is uncorrelated with both $w(k)$ and $v(k)$ and has the following properties:

(1) $E\{x(0)\}=E\{w(0)\}=E\{v(0)\}=0, E\left\{x(0) x^{T}(0)\right\}=$ $P(0)=\left[\begin{array}{cc}\widetilde{P}(0) & 0 \\ 0 & \widetilde{P}(0)\end{array}\right]$,

(2) $E\left\{v(k) v^{T}(k)\right\}=\left[\begin{array}{cc}\bar{\gamma}_{0} & 0 \\ 0 & \left(1-\bar{\gamma}_{0}\right) \bar{\gamma}_{1}\end{array}\right]:=V$,

(3) $w(k)$ and $v(k)$ are mutually independent,

(4) $E\left\{C_{\gamma(k)}\right\}=\left[\bar{\gamma}_{0} \widetilde{C}\left(1-\bar{\gamma}_{0}\right) \bar{\gamma}_{1} \widetilde{C}\right]:=\bar{C}$.

Remark 5. Mode (11) properly represents the unreliable networked communication where the time delay and packet dropouts are unavoidable, and the mode is based on the rationale that the induced data latency from the sensor to the controller is restricted not to exceed the sampling period. We can see that if $\gamma_{1}(k)=1$, that is, $y(k)=\gamma_{0}(k) y^{q}(k)+$ $\left(1-\gamma_{0}(k)\right) y^{q}(k-1)$, it denotes randomly varying packet delay, which was first introduced in [25], and subsequently it has been studied in paper [22]. And if $\gamma_{0}(k)=1$, that is, $y(k)=y^{q}(k)$, then there is no packet delay and loss. While $\gamma_{0}(k)=0$ and $\gamma_{1}(k)=0$ there is no packet received by the estimator site; that is, the packet is lost. Also, mode (11) also can be regarded as a special case of paper [26].

In this paper, for systems (13) and (14), the full-order filter considered is described by

$$
\widehat{x}(k+1)=G \widehat{x}(k)+K(y(k)-\bar{C} \widehat{x}(k)),
$$

where $\widehat{x}(k) \in R^{2 n}$ stands for the state estimate of the stochastic systems (13) and (14) and the constant matrices $G$ and $K$ are filter parameters to be determined. follows

Define the steady-state estimation error covariance as

$$
\begin{gathered}
\widehat{X}_{e e}:=\lim _{k \rightarrow \infty} \widehat{X}_{e e}(k):=\lim _{k \rightarrow \infty} E\left\{e(k) e^{T}(k)\right\}, \\
e(k)=x(k)-\widehat{x}(k) .
\end{gathered}
$$

The objective of this paper is to determine the filter parameters $G$ and $K$ such that, for communication limitations including the three aspects previously analyzed. (1) the state of compact system (13) is mean square bounded and (2) the steady-state error covariance $\widehat{X}_{e e}$ of the sequence $\widehat{X}_{e e}(k)$ satisfies

$$
\left[\widehat{X}_{e e}\right]_{i i} \leq \alpha_{i}^{2}, \quad i=1,2, \ldots, 2 n,
$$

where $\left[\widehat{X}_{e e}\right]_{i i}$ means the steady-state variance of the $i$ th error state and $\alpha_{i}^{2}(i=1,2, \ldots, 2 n)$ denotes the prespecified steadystate error-estimation variance constraint on the $i$ th state.

Remark 6. In (18), individual upper bound constraint, which can be obtained according to the engineering requirement, is imposed on individual steady-state estimation error variance. Note that conventional minimum variance filtering method aims to minimize a weighted scalar sum of the estimation error variances and is therefore not able to ensure that the multiple variance requirements will be satisfied [27]. 


\section{Some Preliminary Results}

In this section, we will first establish the state-space model of augmented system followed from compact systems (13) and (14) and filter (16) and then give the upper bound of the variance of the state estimation error under the three aspects of communication limitations.

Define the estimation error by

$$
e(k)=x(k)-\widehat{x}(k) \text {. }
$$

Then, from (14) and (16), we have

$$
\begin{aligned}
y(k)-\bar{C} \hat{x}(k)= & \left(C_{\gamma(k)}+\Delta C_{\gamma(k)}(k)\right) x(k) \\
& +(D+\Delta D(k)) v(k)-\bar{C} \widehat{x}(k) \\
= & \left(C_{\gamma(k)}-\bar{C}\right) x(k)+\bar{C} e(k) \\
& +\Delta C_{\gamma(k)}(k) x(k)+(D+\Delta D(k)) v(k),
\end{aligned}
$$

and, subsequently,

$$
\begin{aligned}
e(k+1)= & (A-G) x(k)+(G-K \bar{C}) e(k) \\
& -\left(K\left(C_{\gamma(k)}-\bar{C}\right)+K \Delta C_{\gamma(k)}(k)\right) x(k) \\
& +B w(k)-K(D-\Delta D(k)) v(k)
\end{aligned}
$$

Again, we define an augmented system, whose state is defined as

$$
\eta(k)=\left[\begin{array}{c}
x(k) \\
e(k)
\end{array}\right]
$$

Then, combining (13) and (21), the dynamics of $\eta(k)$ can be expressed by

$$
\begin{gathered}
\eta(k+1)=(\widehat{A}+\Delta \widehat{A}(k)) \eta(k)+\left(\Phi_{\gamma(k)}+\Delta \Phi_{\gamma(k)}(k)\right) \eta(k) \\
+(\widehat{B}+\Delta \widehat{B}(k)) \widehat{w}(k) \\
e(k)=\left[\begin{array}{ll}
0 & I
\end{array}\right] \eta(k),
\end{gathered}
$$

where $\Delta \widehat{A}(k)=\widehat{H} F(k) \widehat{E}_{1}, \Delta \Phi_{\gamma(k)}(k)=\widehat{H} F(k) E_{2, \gamma(k)}$, and $\Delta \widehat{B}(k)=\widehat{H} F(k) \widehat{E}_{3}$, where

$$
\begin{gathered}
\widehat{A}=\left[\begin{array}{cc}
A & 0 \\
A-G & G-K \bar{C}
\end{array}\right], \quad \Phi_{\gamma(k)}=\left[\begin{array}{cc}
0 & 0 \\
-K\left(C_{\gamma(k)}-\bar{C}\right) & 0
\end{array}\right], \\
\widehat{B}=\left[\begin{array}{cc}
B & 0 \\
B & -K D
\end{array}\right], \\
\widehat{H}=\left[\begin{array}{c}
0 \\
-K \Lambda
\end{array}\right], \quad \widehat{E}_{1}=\left[\begin{array}{ll}
\bar{C} & 0
\end{array}\right],
\end{gathered}
$$

$$
\begin{gathered}
E_{2, \gamma(k)}=\left[\begin{array}{cc}
C_{\gamma(k)}-\bar{C} & 0
\end{array}\right], \quad \widehat{E}_{3}=\left[\begin{array}{ll}
0 & D
\end{array}\right], \\
\widehat{w}(k)=\left[\begin{array}{c}
w(k) \\
v(k)
\end{array}\right] .
\end{gathered}
$$

It is clear that augmented system (23) comprises not only uncertainties but also stochastic matrix sequences $\Phi_{\gamma(k)}$ and $E_{2, \gamma(k)}$. This makes the augmented system (23) a stochastic parameter system, which reflects the characteristic of limited communication capacity. Now, we denote the state covariance matrix of augmented system (23) by

$$
\begin{aligned}
\widehat{X}(k):=E\left\{\eta(k) \eta^{T}(k)\right\} & =E\left\{\left[\begin{array}{c}
x(k) \\
e(k)
\end{array}\right]\left[x^{T}(k) e^{T}(k)\right]\right\} \\
& =\left[\begin{array}{ll}
\widehat{X}_{x x}(k) & \widehat{X}_{x e}(k) \\
\widehat{X}_{e x}(x) & \widehat{X}_{e e}(k)
\end{array}\right] .
\end{aligned}
$$

Since augmented system (23) involves both uncertainties and stochastic matrix sequences $\Phi_{\gamma(k)}$ and $E_{2, \gamma(k)}$ caused by limit communication capacity, the computation of state covariance matrix is complex because of the complexity of the statistics analysis. Hence, the Lyapunov equation that governs the evolution of the covariance matrix is given by the following.

Lemma 7. The evolution Lyapunov equation of the covariance matrix $\widehat{X}(k)$ from (23) can be written as

$$
\begin{aligned}
\widehat{X}(k+1)= & (\widehat{A}+\Delta \widehat{A}(k)) \widehat{X}(k)(\widehat{A}+\Delta \widehat{A}(k))^{T} \\
& +(\widehat{\Phi}+\Delta \widehat{\Phi}(k)) \widehat{X}(k)(\widehat{\Phi}+\Delta \widehat{\Phi}(k))^{T} \\
& +(\widehat{B}+\Delta \widehat{B}(k)) \widehat{W}(k)(\widehat{B}+\Delta \widehat{B}(k))^{T},
\end{aligned}
$$

where $\widehat{A}, \Delta \widehat{A}(k), \widehat{B}, \Delta \widehat{B}(k), \widehat{H}, \widehat{E}_{1}$, and $\widehat{E}_{3}$ satisfy (3) and $\Delta \widehat{\Phi}=$ $\widehat{H} F(k) \widehat{E}_{2}$, where

$$
\begin{gathered}
\widehat{\Phi}=\left[\begin{array}{cc}
0 & 0 \\
-K \widehat{C} & 0
\end{array}\right], \quad \widehat{E}_{2}=\left[\begin{array}{ll}
\widehat{C} & 0
\end{array}\right], \quad \widehat{W}=\left[\begin{array}{cc}
I_{m} & 0 \\
0 & V
\end{array}\right], \\
\widehat{C}=\left[\begin{array}{ll}
\sigma_{\gamma_{0}} \widetilde{C} & \sigma_{c} \widetilde{C}
\end{array}\right], \quad \sigma_{\gamma_{0}}^{2}=\left(1-\bar{\gamma}_{0}\right) \bar{\gamma}_{0}, \\
\sigma_{c}^{2}=\bar{\gamma}_{1}\left(1-\bar{\gamma}_{0}\right)\left(1+\bar{\gamma}_{0} \bar{\gamma}_{1}-\bar{\gamma}_{1}\right) .
\end{gathered}
$$

Proof. For the sake of convenience of discussion, we introduce the following new stochastic sequences:

$$
\begin{gathered}
a(k)=\gamma_{1}(k)-\bar{\gamma}_{1}, \quad b(k)=\gamma_{0}(k) \gamma_{1}(k)-\bar{\gamma}_{0} \bar{\gamma}_{1}, \\
c(k)=a(k)-b(k),
\end{gathered}
$$




$$
\begin{aligned}
& C-\bar{C}=\left[\left(\gamma_{0}(k)-\bar{\gamma}_{0}\right) \widetilde{C}\left(\left(\gamma_{1}(k)-\bar{\gamma}_{1}\right)-\left(\gamma_{0}(k) \gamma_{1}(k)-\bar{\gamma}_{0} \bar{\gamma}_{1}\right)\right) \widetilde{C}_{k-1}\right] \\
& =\left[\left(\gamma_{0}(k)-\bar{\gamma}_{0}\right) \widetilde{C}_{k} c(k) \widetilde{C}_{k-1}\right] \text {. }
\end{aligned}
$$

From Assumption 3, we have the statistical properties relating to stochastic variables $\gamma_{0}(k)-\bar{\gamma}_{0}, a_{k}, b_{k}$, and $c_{k}$ as follows:

$$
\begin{gathered}
E\left\{\left(\gamma_{0}(k)-\bar{\gamma}_{0}\right)^{2}\right\}=\left(1-\bar{\gamma}_{0}\right) \bar{\gamma}_{0}:=\sigma_{\gamma_{0}}^{2}, \\
E\{a(k)\}=0, \\
E\left\{a^{2}(k)\right\}=E\left\{\left(\gamma_{1}(k)-\bar{\gamma}_{1}\right)^{2}\right\} \\
=\left(1-\bar{\gamma}_{1}\right) \bar{\gamma}_{1}:=\sigma_{\gamma_{1}}^{2}, \\
E\{b(k)\}=0, \quad E\left\{b^{2}(k)\right\}=\bar{\gamma}_{0} \bar{\gamma}_{1}\left(1-\bar{\gamma}_{0} \bar{\gamma}_{1}\right), \\
E\{a(k) b(k)\}=\bar{\gamma}_{0} \bar{\gamma}_{1}\left(1-\bar{\gamma}_{1}\right), \\
E\left\{c^{2}(k)\right\}=\bar{\gamma}_{1}\left(1-\bar{\gamma}_{0}\right)\left(1+\bar{\gamma}_{0} \bar{\gamma}_{1}-\bar{\gamma}_{1}\right):=\sigma_{c}^{2} .
\end{gathered}
$$

Now, in order to find the evolution Lyapunov equation of $\widehat{X}(k)$ from augmented system (23), we have to solve two problems: (1) $E\left\{\left(\Phi_{\gamma(k)}+\Delta \Phi_{\gamma(k)} k\right) \widehat{X}(k)\left(\Phi_{\gamma(k)}+\Delta \Phi_{\gamma(k)}(k)\right)^{T}\right\}$ and (2) $E\{\widehat{w}(k) \widehat{w}(k)\}$.

(1) For $E\left\{\left(\Phi_{\gamma(k)}+\Delta \Phi_{\gamma(k)} k\right) \widehat{X}(k)\left(\Phi_{\gamma(k)}+\Delta \Phi_{\gamma(k)}(k)\right)^{T}\right\}$, we have

$$
\begin{aligned}
E\left\{\left(\Phi_{\gamma(k)}+\Delta \Phi_{\gamma(k)} k\right) \widehat{X}(k)\left(\Phi_{\gamma(k)}+\Delta \Phi_{\gamma(k)}(k)\right)^{T}\right\} \\
=E\left\{\Phi_{\gamma(k)} \widehat{X}(k) \Phi_{\gamma(k)}^{T}\right\}+E\left\{\Phi_{\gamma(k)} \widehat{X}(k) \Delta \Phi_{\gamma(k)}^{T}(k)\right\} \\
+E\left\{\Delta \Phi_{\gamma(k)}(k) \widehat{X}(k) \Phi_{\gamma(k)}^{T}\right\} \\
+E\left\{\Delta \Phi_{\gamma(k)}(k) \widehat{X}(k) \Delta \Phi_{\gamma(k)}^{T}(k)\right\} .
\end{aligned}
$$

(i) For the first expression on the right hand of the above equation,

$E\left\{\Phi_{\gamma(k)} \eta(k) \eta^{T}(k) \Phi_{\gamma(k)}^{T}\right\}$

$=E\left\{\left[\begin{array}{cc}0 & 0 \\ -K(C-\bar{C}) & 0\end{array}\right]\left[\begin{array}{cc}\widehat{X}_{x x} & \widehat{X}_{x e} \\ \widehat{X}_{x e}^{T} & \widehat{X}_{e e}\end{array}\right]\left[\begin{array}{cc}0 & -(C-\bar{C})^{T} K^{T} \\ 0 & 0\end{array}\right]\right\}$

$=\left[\begin{array}{lc}0 & 0 \\ 0 & E\left\{K(C-\bar{C}) \widehat{X}_{x x}(C-\bar{C})^{T} K^{T}\right\}\end{array}\right]$.
Noticing that $\widehat{X}_{x x}(k)=\operatorname{diag}\{\widetilde{X}(k) \widetilde{X}(k-1)\}$, where $\widetilde{X}(k):=E\left\{\widetilde{x}(k) \widetilde{x}^{T}(k)\right\}$, we have

$$
\begin{aligned}
E & \left\{\left(C_{k}-\bar{C}_{k}\right) \widehat{X}_{x x}\left(C_{k}-\bar{C}_{k}\right)^{T}\right\} \\
& =E\left\{\left(\gamma_{0}(k)-\bar{\gamma}_{0}\right)^{2}\right\} \widetilde{C} \widetilde{X}(k) \widetilde{C}^{T}+E\left\{c^{2}(k)\right\} \widetilde{C} \widetilde{X}_{k-1} \widetilde{C}^{T} \\
& =\sigma_{\gamma_{0}}^{2} \widetilde{C} \widetilde{X}(k) \widetilde{C}^{T}+\sigma_{c}^{2} \widetilde{C} \widetilde{X}(k+1) \widetilde{C}^{T} \\
& =\left[\begin{array}{lll}
\sigma_{\gamma_{0}} \widetilde{C}_{k} & \sigma_{c} \widetilde{C}_{k-1}
\end{array}\right] \widehat{X}_{x x}\left[\begin{array}{lll}
\sigma_{\gamma_{0}} \widetilde{C}_{k} & \sigma_{c} \widetilde{C}_{k-1}
\end{array}\right]^{T} \\
& =\widehat{C}_{X_{x x}} \widehat{C} .
\end{aligned}
$$

So,

$$
E\left\{\Phi_{\gamma(k)} \eta(k) \eta^{T}(k) \Phi_{\gamma(k)}^{T}\right\}=\widehat{\Phi} \widehat{X}(k) \widehat{\Phi}^{T} .
$$

(ii) Similarly, for the other expressions on the right hand of (32), we obtain that

$$
\begin{aligned}
E\left\{\Phi_{\gamma(k)} \widehat{X}(k) \Delta \Phi_{\gamma(k)}^{T}(k)\right\} & =\widehat{\Phi} \widehat{X}(k) \Delta \widehat{\Phi}(k), \\
E\left\{\Delta \Phi_{\gamma(k)}(k) \widehat{X}(k) \Phi_{\gamma(k)}^{T}\right\} & =\Delta \widehat{\Phi}(k) \widehat{X}(k) \widehat{\Phi}, \\
E\left\{\Delta \Phi_{\gamma(k)}(k) \widehat{X}(k) \Delta \Phi_{\gamma(k)}^{T}(k)\right\} & =\Delta \widehat{\Phi}(k) \widehat{X}(k) \Delta \widehat{\Phi}(k) .
\end{aligned}
$$

Hence, we have

$$
\begin{gathered}
E\left\{\left(\Phi_{\gamma(k)}+\Delta \Phi_{\gamma(k)}^{T}(k)\right) \widehat{X}(k)\left(\Phi_{\gamma(k)}+\Delta \Phi_{\gamma(k)}^{T}(k)\right)^{T}\right\} \\
=(\widehat{\Phi}+\Delta \widehat{\Phi}(k)) \widehat{X}(k)(\widehat{\Phi}+\Delta \widehat{\Phi}(k))^{T} .
\end{gathered}
$$

(2) For $\widehat{W}=E\left\{\widehat{w}(k) \widehat{w}^{T}(k)\right\}$, from the statistical properties of $w(k)$ and $v(k)$, it is easy to obtain that

$$
\widehat{W}=\left[\begin{array}{c}
w(k) \\
v(k)
\end{array}\right]\left[\begin{array}{ll}
w^{T}(k) & v^{T}(k)
\end{array}\right]=\left[\begin{array}{cc}
I_{m} & 0 \\
0 & V
\end{array}\right] .
$$

Consequently, (27) holds. This completes the proof of this lemma.

Define the steady-state covariance $\widehat{X}$ of (27) as follows:

$$
\widehat{X}:=\lim _{k \rightarrow \infty} \widehat{X}(k)=\left[\begin{array}{cc}
\widehat{X}_{x x} & \widehat{X}_{x e} \\
\widehat{X}_{x e}^{T} & \widehat{X}_{e e}
\end{array}\right] .
$$

Then, from $[7,22,28]$, we have the following useful lemma. 
Lemma 8. There exists a unique symmetric positive semidefinite solution to the following discrete-time equation:

$$
\begin{aligned}
\widehat{X}= & (\widehat{A}+\Delta \widehat{A}) \widehat{X}(\widehat{A}+\Delta \widehat{A})^{T}+(\widehat{\Phi}+\Delta \widehat{\Phi}) \widehat{X}(\widehat{\Phi}+\Delta \widehat{\Phi})^{T} \\
& +(\widehat{B}+\Delta \widehat{B}(k)) \widehat{W}(k)(\widehat{B}+\Delta \widehat{B}(k))^{T} ;
\end{aligned}
$$

that is, the convergence of $\widehat{X}(k)$ in (27) is guaranteed to a constant value $\widehat{X}$, and the state of (23) is mean square bounded if and only if

$$
\rho\left\{(\widehat{A}+\Delta \widehat{A}) \otimes(\widehat{A}+\Delta \widehat{A})^{T}+(\widehat{\Phi}+\Delta \widehat{\Phi}) \otimes(\widehat{\Phi}+\Delta \widehat{\Phi})\right\}<1,
$$

where $\rho$ is the spectral radius and $\otimes$ is the Kronecker product.

\section{Main Results and Proofs}

The main results are presented in this section. To start with, we first recall some lemmas that will be needed in the proof of our main results.

Lemma 9 (see [29]). Given matrices $A, H, E$, and $F$ with compatible dimensions such that $F F^{T} \leq I$, let $X$ be a positive definite matrix and $\alpha>0$ an arbitrary constant such that $\alpha^{-1} I-E X E^{T}>0$. Then, we have

$$
\begin{aligned}
& (A+H F E) X(A+H F E)^{T} \\
& \quad \leq A\left(X^{-1}-\alpha E^{T} E\right)^{-1} A^{T}+\alpha^{-1} H H^{T} .
\end{aligned}
$$

Lemma 10 (see [30]). Given matrices $\Xi \in R^{s \times r}$ and $\Theta \epsilon$ $R^{s \times t}$, there exists a solution $X \in R^{r \times t}$ to the matrix equation $\Xi X=\Theta$ if and only if $\left(I-\Xi \Xi^{+}\right) \Theta=0$, where $\Xi^{+}$denotes the Moore-Penrose inverse of $\Xi$. Furthermore, all solutions can be parameterized by

$$
X=\Xi^{+} \Theta+\left(I-\Xi^{+} \Xi\right) Z,
$$

where $Z \in R^{r \times t}$ is an arbitrary matrix.

Lemma 11 (see [31]). For a given negative definite matrix $\Pi<$ $0\left(\Pi \in R^{2 n \times 2 n}\right)$, there always exists a matrix $L \in R^{2 n \times m}(m \leq$ $2 n)$ such that $\Pi+L L^{T}<0$.

Lemma 12 (Schur complement). Given constant matrices $\Omega_{1}$, $\Omega_{2}$, and $\Omega_{3}$, where $\Omega_{1}=\Omega_{1}^{T}$ and $0<\Omega_{2}=\Omega_{2}^{T}$, then $\Omega_{1}+$ $\Omega_{3}^{T} \Omega_{2}^{-1} \Omega_{3}<0$ if and only if

$$
\left[\begin{array}{cc}
\Omega_{1} & \Omega_{3}^{T} \\
\Omega_{3} & -\Omega_{2}
\end{array}\right]<0 \quad \text { or } \quad\left[\begin{array}{cc}
-\Omega_{2} & \Omega_{3} \\
\Omega_{3}^{T} & \Omega_{1}
\end{array}\right]<0 .
$$

Here, in the following theorem, a two-step approach will be developed to solve the filer problem. Firstly, we will characterize an upper bound on the steady-state error covariance $\widehat{X}$ satisfying (40) in terms of some free parameters, let this upper bound meet prespecified variance constraints (18), and then we parameterize all desired filter gains with which the resulting steady-state error covariance is not more than the obtained upper bound. It is shown that the design of varianceconstraint robust filtering for uncertain systems with limited communication capacity is related to two quadratic matrix inequalities. For presentation convenience, we first define

$$
\begin{gathered}
\Sigma=(A-G)\left(P^{-1}-\alpha \bar{C}^{T} \bar{C}\right)^{-1}(A-G)^{T}+B B^{T}, \\
\Gamma=A\left(P^{-1}-\alpha \bar{C}^{T} \bar{C}\right)^{-1}, \\
R=\widehat{C}\left(P^{-1}-\beta \widehat{C}^{T} \widehat{C}\right)^{-1} \widehat{C}^{T}+D\left(V^{-1}-\xi D^{T} D\right)^{-1} D^{T} \\
+\left(\alpha^{-1}+\beta^{-1}+\xi^{-1}\right) \Lambda \Lambda^{T}+\bar{C} Q \bar{C}^{T}, \\
\Pi=\Sigma+G Q G^{T}-Q-G Q \bar{C}^{T} R^{-1} \bar{C} Q G^{T} .
\end{gathered}
$$

Theorem 13. Let $\beta>0$, and let $\xi>0$ be given positive scalar sequences and inequalities $\beta^{-1} I-\widehat{C} P \widehat{C}^{T}>0$ and $\xi^{-1} I-D V D^{T}>0$ hold. If there exist positive scalars $\alpha>0$, such that the following two quadratic matrix inequalities

$$
\begin{gathered}
A P A^{T}+A P \bar{C}^{T}\left(\alpha^{-1} I-\bar{C} P \bar{C}^{T}\right)^{-1} \bar{C} P A^{T}+B B^{T}-P<0, \\
\Pi=\Sigma+G Q G^{T}-Q-G Q \bar{C}^{T} R^{-1} \bar{C} Q G^{T}<0,
\end{gathered}
$$

respectively, have positive-definite solutions $P>0\left(\alpha^{-1} I-\right.$ $\left.\bar{C} P \bar{C}^{T}>0\right), Q>0$, and

$$
\left(I-\Gamma \Gamma^{+}\right) B B^{T}=0,
$$

where $\Gamma^{+}$denotes the Moore-Penrose inverse of $\Gamma$, and in (50)

$$
G=A+\left(\Gamma^{+} B B^{T}\right)^{T}
$$

Moreover, let $L \in R^{2 n \times m}(m \leq 2 n)$ be an arbitrary matrix satisfying $\Pi+L L^{T}<0$ (see Lemma 11) and $U \in R^{m \times m}$ an arbitrary orthogonal matrix (i.e., $U U^{T}=I$ ). Then, filter (16) with the parameters determined by (51) and

$$
K=G Q \bar{C}^{T} R^{-1}+L U R^{-1 / 2}
$$

will be such that, for all admissible uncertainties caused by the limited communication capacity, (1) the state of augmented system (23) is mean square bounded and (2) the steady-state error covariance $\widehat{X}_{e e}$ meets $\widehat{X}_{e e}<Q$. 
Proof. Define $X:=\operatorname{diag}(P, Q)$. Then, it follows directly from Lemma 9 and definitions (45)-(48) that

$$
\begin{aligned}
(\widehat{A}+ & \Delta \widehat{A}(k)) X(\widehat{A}+\Delta \widehat{A}(k))^{T} \\
& +(\widehat{\Phi}+\Delta \widehat{\Phi}(k)) X(\widehat{\Phi}+\Delta \widehat{\Phi}(k))^{T} \\
& +(\widehat{B}+\Delta \widehat{B}(k)) \widehat{W}(\widehat{B}+\Delta \widehat{B}(k))^{T} \\
\leq & \widehat{A}\left(X^{-1}-\alpha \widehat{E}_{1}^{T} \widehat{E}_{1}\right)^{-1} \widehat{A}^{T}+\widehat{\Phi}\left(X^{-1}-\beta \widehat{E}_{2}^{T} \widehat{E}_{2}\right)^{-1} \widehat{\Phi}^{T} \\
& +\widehat{B}\left(\widehat{W}^{-1}-\xi \widehat{E}_{3}^{T} \widehat{E}_{3}\right)^{-1} \widehat{B}^{T}+\left(\alpha^{-1}+\beta^{-1}+\xi^{-1}\right) \widehat{H} \widehat{H}^{T} \\
& -X:=\Psi:=\left[\begin{array}{ll}
\Psi_{11} & \Psi_{12} \\
\Psi_{12}^{T} & \Psi_{22}
\end{array}\right],
\end{aligned}
$$

where

$$
\begin{aligned}
\Psi_{11}= & A\left(P^{-1}-\alpha \bar{C}^{T} \bar{C}\right)^{-1} A^{T}-P+B B^{T}, \\
\Psi_{12}= & A\left(P^{-1}-\alpha \bar{C}^{T} \bar{C}\right)^{-1}(A-G)^{T}+\overline{B B}^{T}, \\
\Psi_{22}= & (A-G)\left(P^{-1}-\alpha \bar{C}^{T} \bar{C}\right)^{-1}(A-G)^{T} \\
& +(G-K \bar{C}) Q(G-K \bar{C})^{T} \\
& +K \widehat{C}\left(P^{-1}-\beta \widehat{C}^{T} \widehat{C}\right)^{-1} \widehat{C}^{T} K^{T} \\
& +K D\left(V^{-1}-\xi D^{T} D\right)^{-1} D^{T} K^{T} \\
& +\overline{B B}^{T}-Q+\left(\alpha^{-1}+\beta^{-1}+\xi^{-1}\right) K \Lambda \Lambda^{T} K^{T} .
\end{aligned}
$$

Making use of Matrix Inverse Theory, we have

$$
\left(P^{-1}-\alpha E^{T} E\right)^{-1}=P+P E^{T}\left(\alpha^{-1} I-E P E^{T}\right)^{-1} E P
$$

and therefore, from inequality (49), we can obtain that $\Psi_{11}<$ 0 .

Next, notice that the matrix $A$ is singular. It then follows from Lemma 10 that, there exists a solution $G$ such that $\Psi_{12}=$ 0 if and only if (51) holds. Furthermore, if (51) is true, (52) gives a solution. Hence, substituting the expression of $G$ in (52) into (56) leads to $\Psi_{12}=0$ easily.
Furthermore, we now consider $\Psi_{22}$, by using definitions (45)-(48), and we can rearrange (57) as follows:

$$
\begin{aligned}
\Psi_{22}= & \Phi+G Q G^{T}-Q-G Q \bar{C}^{T} K^{T}-K \bar{C} Q G^{T} \\
& +K\left(\widehat{C}\left(P^{-1}-\beta \widehat{C}^{T} \widehat{C}\right)^{-1} \widehat{C}^{T}+D\left(V^{-1}-\xi D^{T} D\right)^{-1} D^{T}\right. \\
& \left.+\left(\alpha^{-1}+\beta^{-1}+\xi^{-1}\right) \Lambda \Lambda^{T}+\bar{C} Q \bar{C}^{T} \widehat{C}\right) K^{T} \\
= & \Phi+G Q G^{T}-Q-G Q \bar{C}^{T} R^{-1} \bar{C} Q G^{T} \\
& +\left(K R^{1 / 2}-G Q \bar{C}^{T} R^{-1 / 2}\right)\left(K R^{1 / 2}-G Q \bar{C}^{T} R^{-1 / 2}\right)^{T} \\
& \quad \Pi+\left(K R^{1 / 2}-G Q \bar{C}^{T} R^{-1 / 2}\right)\left(K R^{1 / 2}-G Q \bar{C}^{T} R^{-1 / 2}\right)^{T} .
\end{aligned}
$$

Noticing the expression of $K$ in (53) and the fact that $U U^{T}=I$, we have

$$
\left(K R^{1 / 2}-G Q \bar{C}^{T} R^{-1 / 2}\right)\left(K R^{1 / 2}-G Q \bar{C}^{T} R^{-1 / 2}\right)^{T}=L L^{T} .
$$

Hence, it follows from (59) and in view of the definition of the matrix $L$ and inequality (50), we can obtain that and the $\Psi_{22}=\Pi+L L^{T}<0$.

To this end, we can conclude that $\Psi<0$. Thus, it follows from (47) that

$$
\begin{aligned}
(\widehat{A}+ & \Delta \widehat{A}(k)) X(\widehat{A}+\Delta \widehat{A}(k))^{T} \\
& -X+(\widehat{\Phi}+\Delta \widehat{\Phi}(k)) X(\widehat{\Phi}+\Delta \widehat{\Phi}(k))^{T} \\
\leq & -(\widehat{B}+\Delta \widehat{B}(k)) \widehat{W}(\widehat{B}+\Delta \widehat{B}(k))^{T}+\Psi<0,
\end{aligned}
$$

which leads to (41). From Lemma 8, we know that the state of augmented system (23) is mean square bounded and there exists a symmetric positive semidefinite solution to (40). Then, the first claim of this theorem is proved.

Furthermore, subtract (40) from (61) to give

$$
\begin{aligned}
(\widehat{A}+\Delta \widehat{A}(k))(X-\widehat{X})(\widehat{A}+\Delta \widehat{A}(k))^{T}-(X-\widehat{X}) \\
+(\widehat{\Phi}+\Delta \widehat{\Phi}(k))(X-\widehat{X})(\widehat{\Phi}+\Delta \widehat{\Phi}(k))^{T} \\
\leq \Psi<0,
\end{aligned}
$$

which indicates again from Lemma 8 that $X-\widehat{X} \geq 0$, and therefore

$$
\widehat{X}_{e e}=[\widehat{X}]_{22} \leq[X]_{22}=Q .
$$

This completes the proof of the theorem.

Remark 14. From all of above discussions, we know that, if the conditions of Theorem 13 are all met and the positive-definite solutions $Q>0$ satisfy

$$
[Q]_{i i} \leq \alpha_{i}^{2}, \quad i=1,2, \ldots, 2 n,
$$


then the design objective of robust filter for uncertain systems with limited communication capacity will be accomplished. It is mentionable that the existence of a positive-definite solution to (49) implies the asymptotical Schur stability of system matrix $A$, and this means that Assumption 2 should hold.

Remark 15. It can be seen, from theory 1, that there exits much explicit freedom, such as the choice of parameter $L$, the orthogonal matrix $U$ for our present design approach. We could use the freedom feature to take the more expected performance constraints into account within the same framework (e.g., the transient requirement and reliability behavior on the filtering process), which provide us with one possible future research direction (see Section 5).

Eventually, by using the Schur Lemma (Lemma 12), we present the main results which simultaneously presented a solution to matrix inequalities (49) and (50) as follows.

Corollary 16. If there exist positive scalars $\alpha>0$ and two positive-definite matrices $P>0, Q>0$ such that the following LMIs (65) and (66) and the matrix inequality (50) hold and $Q$ satisfies $[Q]_{i i} \leq \alpha_{i}^{2}(i=1,2, \ldots, 2 n)$, then filter (16) determined by (52) and (53) will achieve the desired robust filtering performance for uncertain systems with limited communication capacity which is discussed previously. Cosider

$$
\begin{gathered}
{\left[\begin{array}{cc}
A P A^{T}-P+B B^{T} & A P \bar{C}^{T} \\
\bar{C} P A^{T} & -\alpha^{-1} I+\bar{C} P \bar{C}^{T}
\end{array}\right]<0} \\
-\alpha^{-1} I+\bar{C} P \bar{C}^{T}<0
\end{gathered}
$$

We now briefly discuss the solvability of quadratic matrix inequalities (49) and (50), which play a key role in designing the expected filters. Since the parameter $Q$ of (50) is not included in (49), from the previous corollary, for $\alpha>0$ and $P>0$, algebraic matrix inequality (49) is equal to the linear matrix inequalities (LMI) (65)-(66) which can be effectively solved by using LMI toolbox in MATLAB. Then, after $\alpha$ and $P$ are gained and positive scalar sequences $\beta>0$ and $\xi>0$ are given satisfying that $\beta^{-1} I-\widehat{C} P \widehat{C}^{T}>0$ and $\xi^{-1} I-D V D^{T}>0$, matrix inequality (50) becomes a standard Riccati-like matrix inequality for $Q>0$ which can be easily solved in terms of the existed approach.

\section{Numerical Example}

In this section, we demonstrate the theory developed in this paper by means of a simple example. Consider the linear discrete-time system described by (1) with parameters given by

$$
\widetilde{A}=\left[\begin{array}{ll}
0.6 & 0.3 \\
0.2 & 0.3
\end{array}\right], \quad \widetilde{B}=\left[\begin{array}{cc}
0.2 & 0 \\
0 & 0.2
\end{array}\right], \quad \widetilde{C}=\left[\begin{array}{ll}
2 & 0 \\
0 & 2
\end{array}\right] .
$$

Furthermore, we assume the following.

(1) The parameter for the quantizer $f(\cdot)$ is $\rho=1 / 3$; then, $\sigma=0.5$; that is, $\Lambda=0.5$.
(2) The stochastic variable $\gamma_{i}(i \in\{1,2\})$ is Bernoulli distributed white sequences taking values 0 and 1 with $E\left\{\gamma_{0}(k)\right\}=\bar{\gamma}_{0}=0.7$ and $E\left\{\gamma_{1}(k)\right\}=\bar{\gamma}_{1}=0.8$, respectively.

(3) The constant $\beta$ and $\xi$ are given by $\beta=0.2$ and $\xi=$ 0.125 .

(4) The process noise $w_{k}$ and measurement noise $v_{k}$ are zero-mean Gaussian white noise sequences with unity covariance.

The purpose of this example is to design the filter parameters $G$ and $K$ such that, for all admissible perturbations and multiple packet dropouts, augmented system (16) is mean square bounded, and the steady-state error covariance satisfies

$$
\begin{array}{ll}
{\left[\widehat{X}_{e e}\right]_{11} \leq 0.4, \quad\left[\widehat{X}_{e e}\right]_{22} \leq 0.3} \\
{\left[\widehat{X}_{e e}\right]_{33} \leq 0.12,} & {\left[\widehat{X}_{e e}\right]_{44} \leq 0.12 .}
\end{array}
$$

We employ the standard LMI techniques to check the solvability of original matrix inequality (49) for $\alpha>0$ and $P>0$ and solve the standard Riccati-like matrix for $Q$. Therefore, we obtain

$$
\begin{gathered}
\alpha=0.1355, \\
P=\left[\begin{array}{cccc}
1.5305 & -1.3492 & 0.3574 & -0.1255 \\
-1.3492 & 1.9906 & -0.1856 & 0.0911 \\
0.3574 & -0.1856 & 4.8334 & -0.8241 \\
-0.1255 & 0.0911 & -0.8241 & 4.9600
\end{array}\right], \\
Q=\left[\begin{array}{cccc}
0.3873 & 0.0862 & -0.0030 & -0.0057 \\
0.0862 & 0.2988 & -0.0019 & -0.0036 \\
-0.0030 & -0.0019 & 0.1001 & 0.0001 \\
-0.0057 & -0.0036 & 0.0001 & 0.1002
\end{array}\right]
\end{gathered}
$$
follows:

From (52), one of the filter parameters, $G$, is calculated as

$$
G=\left[\begin{array}{cccc}
0.6151 & 0.3092 & -0.0111 & -0.0210 \\
0.2068 & 0.3068 & -0.0064 & -0.0115 \\
1.0000 & 0 & 0 & 0 \\
0 & 1.0000 & 0 & 0
\end{array}\right]
$$

To obtain another parameter $K$, we choose $L$ meeting $\Pi+$ $L L^{T}<0$ and select the orthogonal matrix $U$ as follows:

Case 1: $L=\left[\begin{array}{c}0.4 \\ 0 \\ 0.4 \\ 0\end{array}\right], \quad U=1$,

$$
\text { Case } 2: L=\left[\begin{array}{c}
0.1 \\
0 \\
0.1 \\
0
\end{array}\right], \quad U=-1 \text {. }
$$




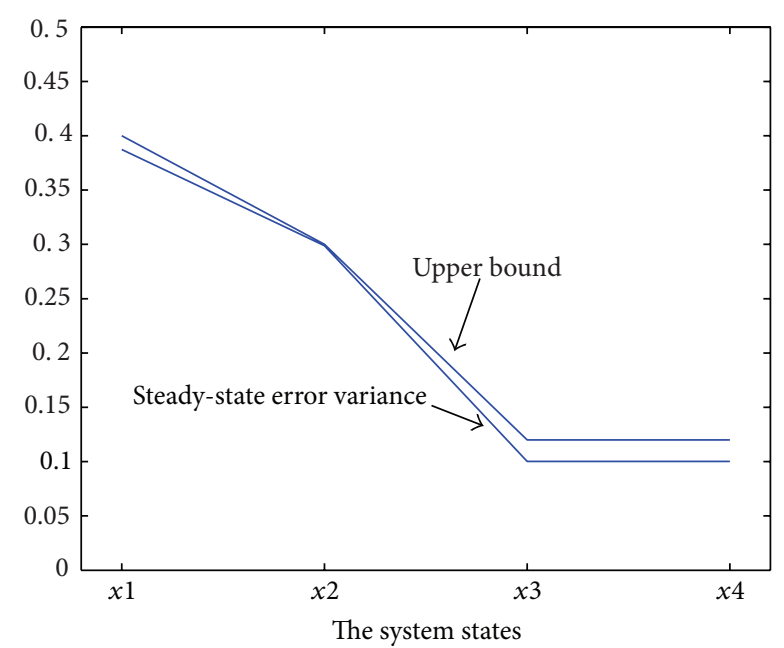

FIGURE 1: Steady-state error variance and the individual upper bound.

Then, we can get the parameter $K$ from (53) as follows:

$$
\begin{aligned}
& \text { Case 1: } K=\left[\begin{array}{l}
0.2418 \\
0.0454 \\
0.2552 \\
0.0811
\end{array}\right], \\
& \text { Case 2: } K=\left[\begin{array}{l}
0.0473 \\
0.0454 \\
0.0607 \\
0.0811
\end{array}\right] .
\end{aligned}
$$

From the above simulated results, it is not difficult to get that $P>0$ and $Q>0$; hence, the specified mean square bounded is achieved. Furthermore, in Figure 1, the above curve is the bound and the under curve is the steadystate error variance; it is obvious that the steady-state error variance is not more than the individual upper bound, which verifies that steady-state error variance constraint is also achieved. Therefore, it is shown that the theory developed in this paper is effective and practical.

\section{Conclusion}

In this paper, we have considered a variance-constraint robust filtering problem for discrete-time systems with limited communication capacity. It has been shown that the problem is solvable if a couple of LMIs or Riccati-like matrix inequalities have positive definite solutions. A numerical example is presented to demonstrate the effectiveness and flexibility of the proposed design approach.

Further extension of the present results to more complex systems such as nonlinear systems is a possible future research direction. Moreover, in NCSs, there usually exist simultaneously the parameter uncertainties and intermediate uncertainties; thus, simultaneously considering delay, quantization, observation losses, and parameter uncertaintes is another future avenue of research.

\section{Conflict of Interests}

The authors declare that there is no conflict of interests regarding the publication of this paper.

\section{Acknowledgments}

This work is supported by Liaoning Province Education Department Scientific Research Project (L2012445), the National Natural Science Foundation of China under Grant no. 60504017, Fok Ying Tung Education Foundation under Grant 111066, and Program for New Century Excellent Talents in University under Grant NCET-04-0982.

\section{References}

[1] M. Moayedi, Y. K. Foo, and Y. C. Soh, "Adaptive Kalman filtering in networked systems with random sensor delays, multiple packet dropouts and missing measurements," IEEE Transactions on Signal Processing, vol. 58, no. 3, pp. 1577-1588, 2010.

[2] W.-A. Zhang, L. Yu, and G. Feng, "Optimal linear estimation for networked systems with communication constraints," Automatica, vol. 47, no. 9, pp. 1992-2000, 2011.

[3] H. J. Gao and T. W. Chen, " $H_{\infty}$ estimation for uncertain systems with limited communication capacity," IEEE Transactions on Automatic Control, vol. 52, no. 11, pp. 2070-2084, 2007.

[4] H. L. Dong, Z. D. Wang, and H. J. Gao, "Robust $\mathrm{H}_{\infty}$ filtering for a class of nonlinear networked systems with multiple stochastic communication delays and packet dropouts," IEEE Transactions on Signal Processing, vol. 58, no. 4, pp. 1957-1966, 2010.

[5] S. Sun, "Linear minimum variance estimators for systems with bounded random measurement delays and packet dropouts," Signal Processing, vol. 89, no. 7, pp. 1457-1466, 2009.

[6] Z. D. Wang, F. W. Yang, D. W. C. Ho, and X. Liu, "Robust finite-horizon filtering for stochastic systems with missing measurements," IEEE Signal Processing Letters, vol. 12, no. 6, pp. 437-440, 2005.

[7] Z. D. Wang, D. W. C. Ho, and X. H. Liu, "Variance-constrained filtering for uncertain stochastic systems with missing measurements," IEEE Transactions on Automatic Control, vol. 48, no. 7, pp. 1254-1258, 2003.

[8] S. C. Smith and P. Seiler, "Estimation with lossy measurements: jump estimators for jump systems," IEEE Transactions on Automatic Control, vol. 48, no. 12, pp. 2163-2171, 2003.

[9] K. Gu, V. Kharitonov, and J. Chen, Stability of Time-Delay Systems, Birkhauser, 1st edition, 2003.

[10] L. Schenato, "Optimal estimation in networked control systems subject to random delay and packet loss," in Proceedings of the 45th IEEE Conference on Decision \& Control (CDC '06), pp. 5615-5620, 2006.

[11] M. Fu and L. Xie, "The sector bound approach to quantized feedback control," IEEE Transactions on Automatic Control, vol. 50, no. 11, pp. 1698-1711, 2005.

[12] V. Malyavej and A. V. Savkin, "The problem of optimal robust Kalman state estimation via limited capacity digital communication channels," Systems \& Control Letters, vol. 54, no. 3, pp. 283-292, 2005.

[13] J. Liang, Z. Wang, and X. Liu, "Distributed state estimation for discrete-time sensor networks with randomly varying nonlinearities and missing measurements," IEEE Transactions on Neural Networks, vol. 22, no. 3, pp. 486-496, 2011. 
[14] D. R. Ding, Z. D. Wang, H. Dong, and H. Shu, "Distributed $\mathrm{H}_{\infty}$ state estimation with stochastic parameters and nonlinearities through sensor networks: the finite-horizon case," Automatica, vol. 48, no. 8, pp. 1575-1585, 2012.

[15] B. Wang and G. Guo, "Robust estimation for discrete timevarying systems with limited communication capacity," Asian Journal of Control, vol. 14, no. 2, pp. 502-511, 2012.

[16] F. W. Yang, Z. D. Wang, and Y. S. Hung, "Robust Kalman filtering for discrete time-varying uncertain systems with multiplicative noises," IEEE Transactions on Automatic Control, vol. 47, no. 7, pp. 1179-1183, 2002.

[17] Z. Dong and Z. You, "Finite-horizon robust Kalman filtering for uncertain discrete time-varying systems with uncertaincovariance white noises," IEEE Signal Processing Letters, vol. 13, no. 8, pp. 493-496, 2006.

[18] F. Wang and V. Balakrishnan, "Robust steady-state filtering for systems with deterministic and stochastic uncertainties," IEEE Transactions on Signal Processing, vol. 51, no. 10, pp. 2550-2558, 2003.

[19] E. Yaz and R. E. Skelton, "Continuous and discrete state estimation with error covariance assignment," in Proceedings of the IEEE Conference of Decision Control, pp. 3091-3092, Brighton, UK, 1991.

[20] Z. D. Wang, B. Huang, and P. Huo, "Sampled-data filtering with error covariance assignment," IEEE Transactions on Signal Processing, vol. 49, no. 3, pp. 666-670, 2001.

[21] Z. D. Wang and H. Qiao, "Robust filtering for bilinear uncertain stochastic discrete-time systems," IEEE Transactions on Signal Processing, vol. 50, no. 3, pp. 560-567, 2002.

[22] Z. D. Wang, D. W. C. Ho, and X. H. Liu, "Robust filtering under randomly varying sensor delay with variance constraints," IEEE Transactions on Circuits and Systems II: Express Briefs, vol. 51, no. 6, pp. 320-326, 2004.

[23] N. Elia and S. K. Mitter, "Stabilization of linear systems with limited information," IEEE Transactions on Automatic Control, vol. 46, no. 9, pp. 1384-1400, 2001.

[24] M. Fu and L. Xie, "The sector bound approach to quantized feedback control," IEEE Transactions on Automatic Control, vol. 50, no. 11, pp. 1698-1711, 2005.

[25] E. Yaz and A. Ray, "Linear unbiased state estimation for random models with sensor delay," in Proceedings of the 35th IEEE Conference on Decision and Control, pp. 47-52, Kobe, Japan, 1996.

[26] S. L. Sun, "Linear minimum variance estimators for systems with bounded random measurement delays and packet dropouts," Signal Processing, vol. 89, no. 7, pp. 1457-1466, 2009.

[27] R. F. Stengel, Stochastic Optimal Control: Theory and Application, John Wiley \& Sons, New York, NY, USA, 1986.

[28] W. L. de Koning, "Optimal estimation of linear discrete-time systems with stochastic parameters," Automatica, vol. 20, no. 1, pp. 113-115, 1984.

[29] L. Xie, Y. C. Soh, and C. E. de Souza, "Robust Kalman filtering for uncertain discrete-time systems," IEEE Transactions on Automatic Control, vol. 39, no. 6, pp. 1310-1314, 1994.

[30] A. Ben-Israel and T. N. E. Greville, Generalized Inverses: Theory and Applications, John Wiley \& Sons, New York, NY, USA, 1974.

[31] Z. Wang and B. Huang, "Robust $H_{2} / H_{\infty}$ filtering for linear systems with error variance constraints," IEEE Transactions on Signal Processing, vol. 48, no. 8, pp. 2463-2467, 2000. 


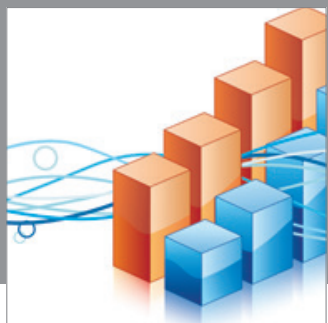

Advances in

Operations Research

mansans

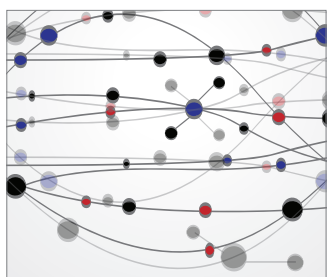

The Scientific World Journal
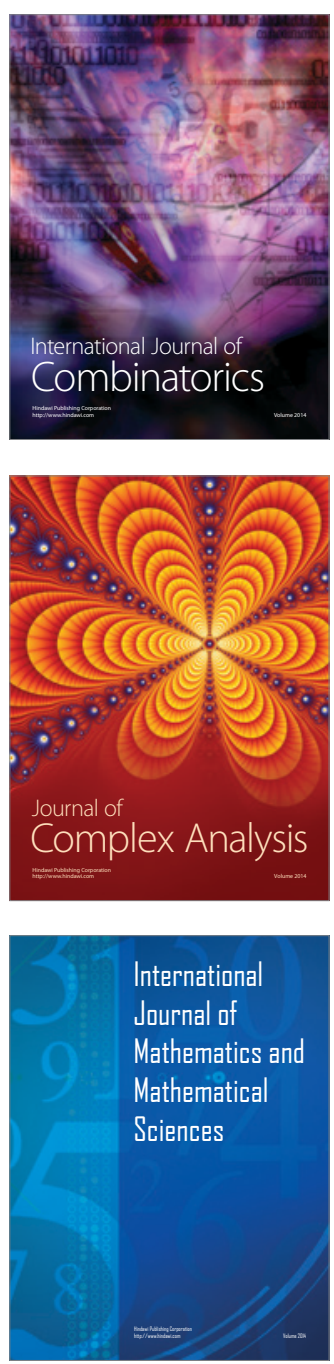
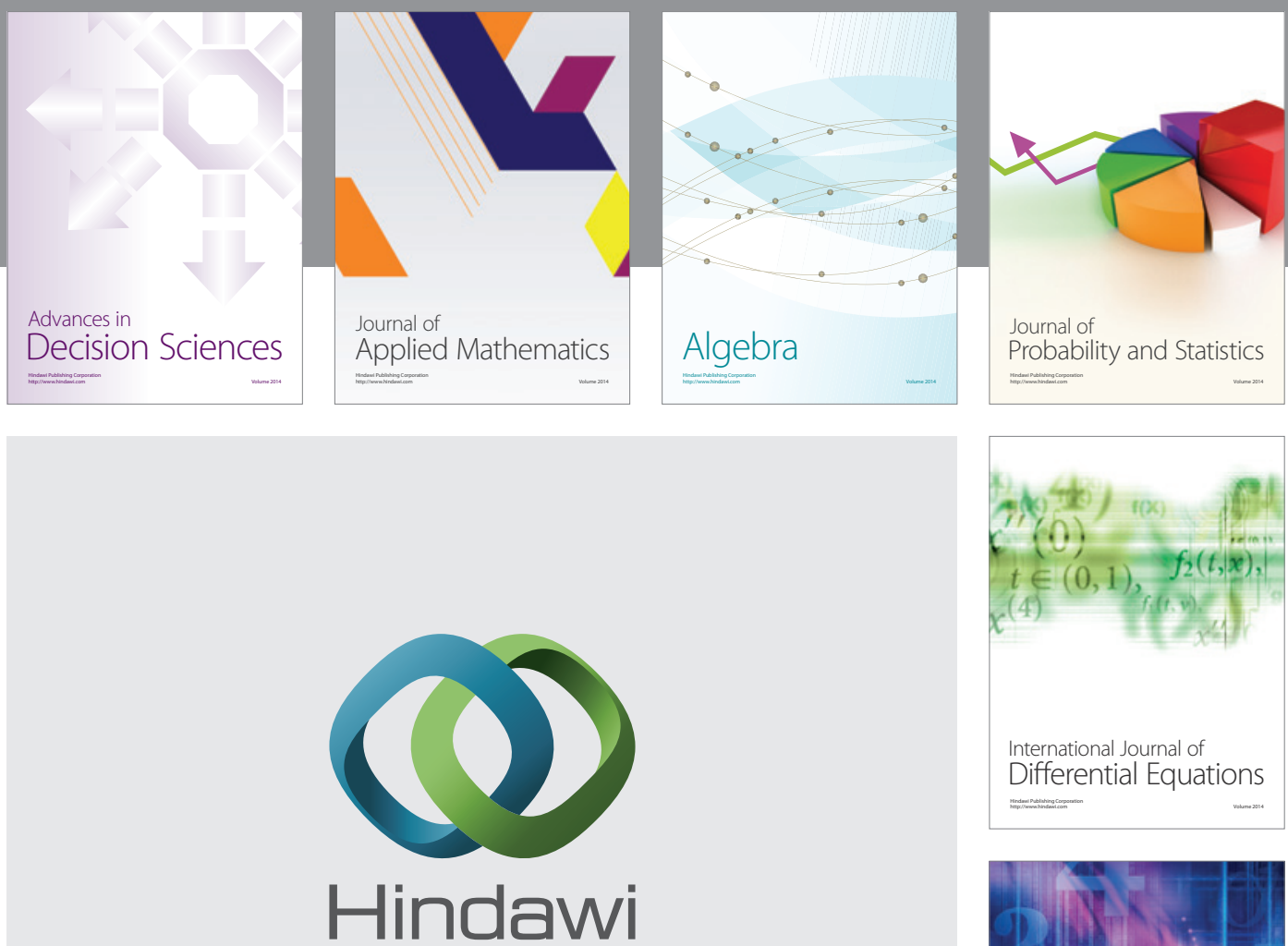

Submit your manuscripts at http://www.hindawi.com
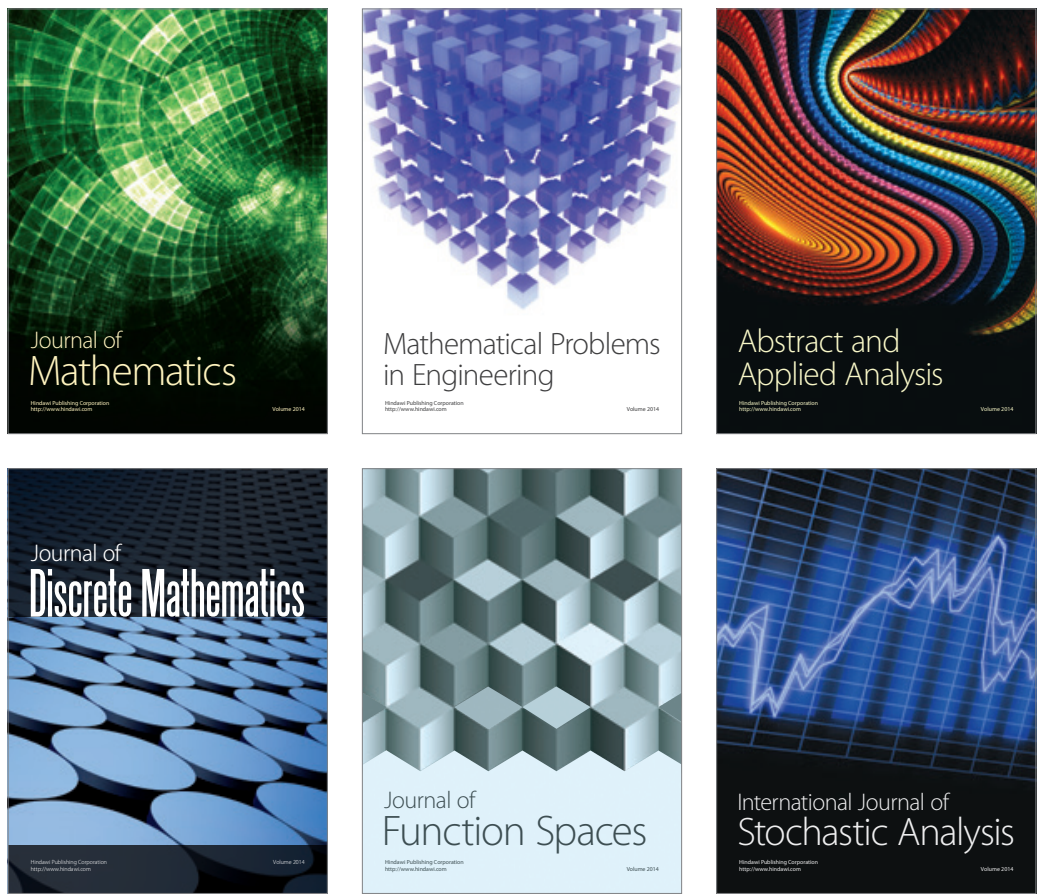

Journal of

Function Spaces

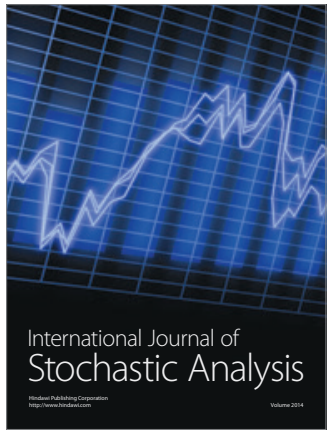

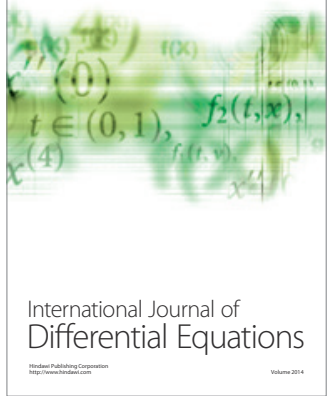
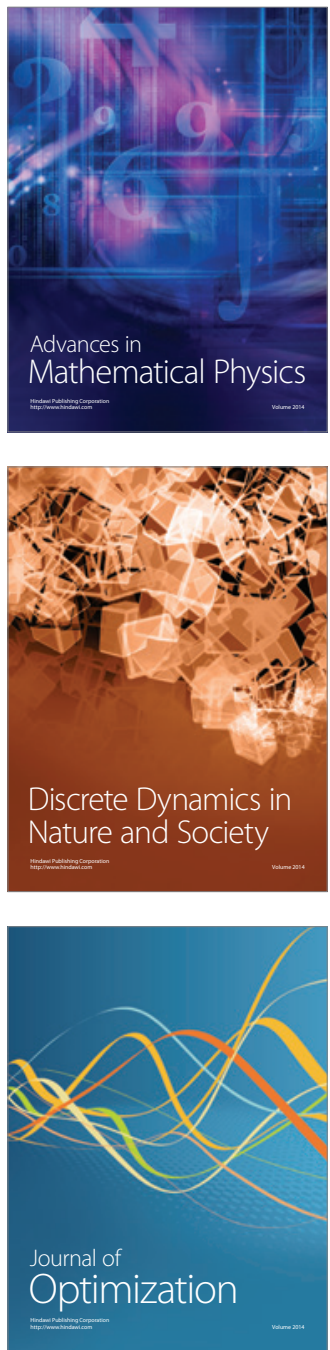